\title{
YourGlove: A Device for Remote Hand Holding
}

\author{
Daniel Gooch \\ Department of Computer Science \\ University of Bath \\ Bath \\ BA2 7AY \\ D.J.Gooch@bath.ac.uk
}

\author{
Leon Watts \\ Department of Computer Science \\ University of Bath \\ Bath \\ BA2 7AY \\ L.Watts@bath.ac.uk
}

\begin{abstract}
Computer mediated communication (CMC) systems, as with the great majority of interactive systems, rely on their users' ability to encode and decode messages in sight and sound. They are however notoriously problematic for implicit communication and fostering a sense of intimacy between couples. We describe the design concept and construction of a device intended to exploit existing behaviour and a haptic sensory channel: hand holding.
\end{abstract}

\section{Social Communication, Affective Interaction, Haptic Interfaces, Remote Touch}

\section{INTRODUCTION}

People care about their social relationships. However, large numbers of people find that they have to carry out these relationships whilst physically separated from their loved ones for extended periods of time. These people have to exploit a variety of communication technologies to help maintain their relationship; however none of these technologies replace the emotional closeness of being with their partner. One of the notable things about mainstream communication media is that none of them leverage haptic communication to transmit information.

As many of the acts involved in intimacy involve touch, consideration of haptics in the panoply of $\mathrm{CMC}$ technologies is an approach worth considering. Several researchers have developed haptic devices to somehow approximate the close physical contact that highly personal relationships often enjoy. Many of the proposed devices start from a completely abstract perspective (e.g. Itoh et al. (2002)), whereas others have taken a familiar artefact and supplemented it with an abstract haptic signal such as a bed (e.g. Goodman and Misilim (2003)).

An alternative approach to designing intimate devices would be to take a familiar social behaviour and selectively model it across a distance through haptic means. The only action which has received even minimal interest is hugging (eg. Mueller et. al, (2005)). Even then, there are implementation issues which could affect the meaningfulness of haptic action-analogue devices, such as their encumbrance or wearability, motor noise, and battery life.

We report our exploration of social behaviour devices by presenting our consideration of a design concept for remote hand holding. We show our design of a pair of robotic hands for intimate communication. These YourGloves are intended to be used for hand-holding across a distance with the intention of supporting mutuality and reciprocity in the execution of mindful physical contact: when robotic hand (a) is held, robotic hand (b) grips and vice-versa.

\section{DESIGN CONCEPT}

The intention of the device is not to replicate hand holding per se. As each YourGlove is clearly similar to a hand, we wanted to avoid the Uncanny Valley implications that replication would entail (Mori, M. 1970). Instead, we wanted the device to use the powerful metaphor of hand holding in a way which mimics the 'normal' act, but does not try to replicate it. By this we mean we have not attempted to make the hand lifelike in terms of having a skin-like cover, body heat or complex mechanical joints; it is only the behaviour that is being replicated.

\section{CONTEXT OF USE}

Use of the device is possibly constrained by the need for an additional communication medium. We anticipate two basic situations in which YourGlove 
might play a role. The first is when, at a predetermined time, a couple are chatting, over email or IM or VOIP, and feel the need to share a tactile experience. The second is when a participant is using their computer and wishes to hold hands on the expectation or hope that their partner is there. The possibility of use has, deliberately, been left up to the users to form their own pattern of use.

\section{DEVICE DESIGN}

The hand mechanism is constructed as a modified robotic hand ${ }^{1}$, with electric motors and computer control. The digits of the hand are constructed from sections of flexible plastic ducting, relieved by cuts that correspond to the physical arrangement of joints in the human hand. The grip of the hand is operated by means of strings that pass though each digit to a fixing point at the 'nail' and are collected within the arm section to a common pulley. The collection of tubes (finger analogues) is covered by a soft fleece glove. Holding the gloved hand creates a moderately realistic impression of holding a 'live' human hand: the combination of semi-rigid internal fingers and padded, resilient fleece conveys an anatomical impression metaphor. Furthermore, the heat of the human hand is insulated by the glove so after a short period, YourGlove seems to warm in way that approximates to the experience of holding the gloved hand of the other.

This mechanised hand is mounted onto some large diameter rigid plastic tubing which, when covered in a shirt sleeve, conveys the visible likeness of an arm. Furthermore, the shirt sleeve could be replaced by the sleeve of an unwanted but cherished item from a loved ones wardrobe.

The strings operating the hand are motorised by mounting a motor on the side of the arm, putting the spindle through the arm and attaching the strings to the spindle. This is then computer controlled through the use of a Phidget interface board ${ }^{2}$. The motors were specifically selected such that they sound like they are struggling to operate the hand; this should provide a level of reassurance to new users that their hand is not about to be crushed by the mechanised glove. The final device can be seen in Figure 1.

On each hand, a switch is mounted onto the glove and connected to the interface board. Thus, with two YourGloves constructed, holding hand A causes the switch to close and thus hand $B$ to grip. The same is true of hand $B$ to hand $A$. The overall effect is to simulate real-world hand holding.

\footnotetext{
${ }^{1} \mathrm{http}: / /$ www.instructables.com/id/Simple-Animatronics-robotichand/

${ }^{2}$ http://www.phidgets.com/
}

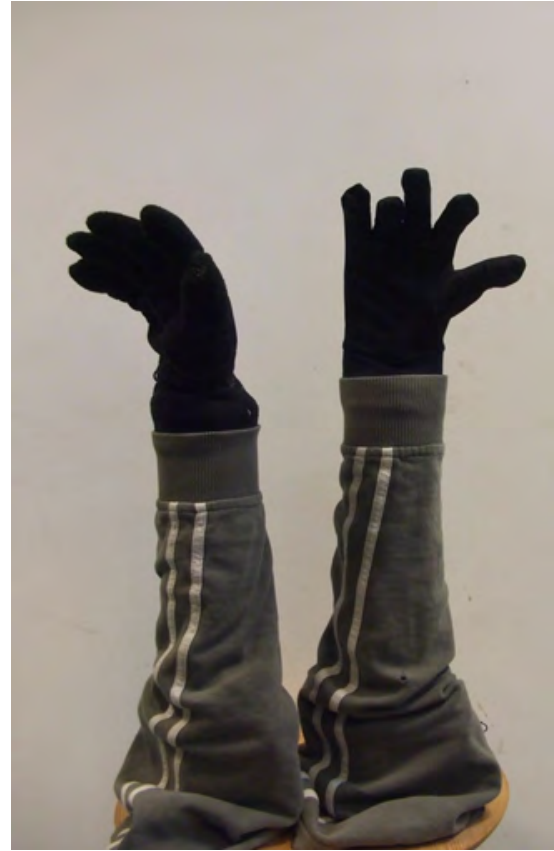

Figure 1: A Pair of YourGloves

\section{FUTURE WORK}

In this paper, we have taken as our point of departure a particular intimate physical act: hand holding. Although other actions could be considered more intimate, hugging has already received some attention and kissing is a hard action to consider.

The system has yet to be formally evaluated although it has attracted many comments from colleagues including 'creepy' and 'weird'. One of the things we wish to investigate is why certain devices which mimic co-located behaviour succeed (e.g. (Mueller et. al, 2005)) whilst others may not.

\section{REFERENCES}

Goodman, E. and Misilim, M., The sensing beds. In Proc. UbiComp 2003 Workshop.

Itoh, Y., Miyajima, A. and Watanabe, T., 'TSUNAGARI' communication: fostering a feeling of connection between family members. CHI '02, ACM Press. 2002. pp. 810-811.

Mori, M., Bukimi no tani, The uncanny valley (K. F. MacDorman T. Minato, Translation). Energy, 7(4), pp. 33-35.

Mueller, F., Vetere, F., Gibbs, M., Kjeldskov, J., Pedell, S. and Howard, S., Hug over a distance. CHI '05, ACM Press. 2005. pp. 1673-1676. 